\title{
Comparison between Ultrasound-Guided and Anatomic Landmark Puncture of the Right Internal Jugular Vein
}

Marcello Fonseca Salgado Filho ${ }^{1 *}$, Phillip Lawall ${ }^{2}$, Kleber Marcharet de Souza ${ }^{3}$, Izabela Palitot ${ }^{4}$, Izabela Magalhaes ${ }^{5}$ and Henrique Vasconcelos ${ }^{6}$

${ }^{1}$ Professor of Anesthesiology, National Institute of Cardiology, Brazil

${ }^{2}$ National Institute of Cardiology, Brazil

${ }^{3}$ Santa Casa de Mato Grosso do Sul, Brazil

${ }^{4}$ Professor of Nurse, Federal University of Juiz de Fora, Brazil

${ }^{5}$ Hospital Felicio Rocho, Brazil

${ }^{6}$ Professor of Medicine, Federal University of Petrolina, Brazil

\begin{abstract}
Introduction: Central venous puncture guided by Ultrasound (US) is considered the technique of choice by the Society of Cardiovascular Anesthesiologists (SCA), and performing central venous puncture without employing US is considered poor medical practice.
\end{abstract}

Methods: After approval by the Research Ethics Committee of the National Institute of Cardiology a randomized clinical trial was conducted electronically with 40 patients (of both genders) who were between 45 and 65 years old and were scheduled to undergo Coronary Artery Bypass Surgery (CABG). The patients were electronically randomized (GraphPad 5.0 OS for Macintosh) divided into two groups of 20: the Ultrasound Group (USG) and the Blind Group (BG). The BG subjects were punctured according to anatomical definitions, and the USG subjects were punctured with US guidance.

Results: There were no differences in the demographic data. The duration of the puncture procedure was equal for the two groups (USG=130.7 $\pm 57.1 \mathrm{sec}$ and $B G=149.4 \pm 98.1 \mathrm{sec} ; \mathrm{p}=0.78$ ). The incidence of carotid puncture was the same for the two groups (USG $=0(0 \%)$ and $B G=2(8.5 \%) ; p=0.48)$. The USG had a lower incidence of changing the puncture site $(\mathrm{USG}=0(0 \%)$ and $\mathrm{BG}=4(23 \%) ; \mathrm{p}=0.03)$ and a higher incidence of catheter implantation in a single attempt (USG=16 (100\%) and $B G=10(58 \%)$.

Conclusions: The use of US for insertion of central venous catheters is associated with a higher incidence of performing venous puncture in a single attempt, a lower incidence of vascular complications and a lower incidence of changing the puncture site.

Keywords: Ultrasonography; Anatomic landmark; Central venous catheters; Coronary artery bypass surgery

\section{Introduction}

In the United States of America, more than 5 million central venous catheters are placed each year for various reasons, including hemodynamic monitoring, fluid and drug administration, and hemodialysis [1,2].

Despite frequent training and practice, insertion of a vascular catheter is associated with complications that may vary between $5 \%$ and 19\% (arterial puncture, pneumothorax, hemothorax, air embolism, catheter embolism, and cardiac arrhythmias among others) [1-3]. These complications usually occur when doctors have little experience or due to anatomical abnormalities (such as obesity, cachexia, tumors, vascular thrombosis, and congenital anomalies), emergency situations, and the presence of comorbidities (emphysema and coagulopathies) $[2]$.

Ultrasound (US) was introduced to clinical practice at the beginning of 1970 and is currently indicated for various clinical situations [2]. In 2012, Troianos et al. described the use of US for location and cannulation of the right jugular vein [2].

US-guided central venous puncture is considered the technique of choice, with a classification of " $1 \mathrm{~A}$ " by the Society of Cardiovascular Anesthesiologists (SCA) [2]. The Agency for Healthcare Research and Quality recommends US for the insertion of central venous catheters as one of the 11 good medical practices in patient care $[2,4]$.

However, according to a study published by the SCA, $67 \%$ of anesthesiologists who are members of this society either do not use or almost never use US-guided puncture, and only $15 \%$ routinely use US [5]. One of the primary reasons for not routinely using US is equipment unavailability, lack of theoretical/practical training, and practicality concerns regarding the technique $[2,4]$.

US-guided central venous puncture also yields such complications as carotid puncture $(75 \%)$, carotid injury $(3 \%)$, stroke $(1 \%)$, and hemothorax (4\%) [2]. In addition to these complications, the learning curve is greater than that for blind puncture, which increases the time necessary to perform US-guided puncture. The difficulty of obtaining the proper equipment for performing central venous puncture is another challenge hindering the routine use of this technique $[5,6]$.

The goal of the present study was to compare US-guided and blind puncture of the Right Internal Jugular Vein (RIJV).

*Corresponding author: Marcello Fonseca Salgado Filho, Master, Professor of Anesthesiology, National Institute of Cardiology, Rua sao luiz, 281 CEP 36039-010, Brazil, Tel: 55(32)99858833; Fax: 55(32)99858833; E-mail mfonsecasalgado@hotmail.com

Received September 20, 2013; Accepted October 26, 2013; Published October 30, 2013

Citation: Filho MFS, Lawall P, de Souza KM, Palitot I, Magalhaes I, et al. (2013) Comparison between Ultrasound-Guided and Anatomic Landmark Puncture of the Right Internal Jugular Vein. J Cardiovasc Dis Diagn 1: 128. doi:10.4172/2329 9517.1000128

Copyright: ( 2013 Filho MFS, et al. This is an open-access article distributed under the terms of the Creative Commons Attribution License, which permits unrestricted use, distribution, and reproduction in any medium, provided the original author and source are credited. 


\section{Methods}

After approval by the Human Research Ethics Committee of the National Institute of Cardiology/Ministry of Health, compliance with the ethical standards of the Declaration of Helsinki, an electronically randomized clinical trial (GraphPad 5.0 OS for Macintosh) was performed.

Forty patients of both gender, ages between 45 and 65 years old were scheduled to undergo coronary artery bypass surgery (CABG). The patients were divided into two groups of 20: the Ultrasound Group (USG) and the Blind Group (BG). All of the patients signed an Informed Consent Form (ICF).

Patients meeting any of the following criteria were excluded from the study: 1) ejection fractions lower than $35 \%$; 2) history of emergency procedures; 3 ) history or presence of cardiac arrhythmias; 4) prothrombin time $(\mathrm{PT})<60 \%$ and International Normalized Ratio (INR) $>1.5$; 5) platelet count $<100,000 ; 6)$ Body Mass Index (BMI) $>30$; 7) history of central venous access; 8 ) anatomical changes in the region of the puncture; and 9) refusal to participate in the study.

\section{Radomized technique}

All patients after signed de ICF were electronically randomized (Graph Pad 5.0 OS for Machintosh) divided in two groups: Ultrasound Group (USG) and the Blind Group (BG). Only one cardiovascular anesthesiologist (over ten years of experience in cardiovascular anesthesia) performed all punctures in both groups. The envelopes containing the patient numbers randomized remained in the possession of the other anesthesiologist that was responsible to allocate the patients in US Group or Blind Group to avoid any confounding situation.

\section{Patient preparation}

At the surgical center, each patient was monitored via a two-lead cardioscope (D2 and V5), analysis of the ST segment, pulse oximetry, non-invasive arterial pressure measurements, the bispectral index (BIS) (Datex-Ohmeda ${ }^{\star}$ S/5 Aespire Anesthesia Machine; Helsinki, Finland, 2006), and a precordial stethoscope.

Midazolam $(0.05 \mathrm{mg} / \mathrm{kg})$ was used intravenously as a preanesthetic medication after venoclysis in the upper limb using a Jelco $14 \mathrm{G}$ catheter (Smiths Medical, St. Paul, MN, USA) to infuse $10 \mathrm{ml} / \mathrm{kg}$ lactated Ringer's solution.

After pre-medication, the patient was placed in the dorsal position with the face turned to the left, and the operating table was placed in the Trendelenburg position at approximately 30 degrees.

After positioning the patient, asepsis was performed with disinfecting chlorhexidine and chlorhexidine in alcohol along the entire extension of the right neck and right hemithorax with subsequent placement of sterile surgical drapes.

\section{Technique for blind puncture}

The researcher performed the puncture positioned behind the head of the patient. After dividing the sternocleidomastoid muscle at the medial and lateral portion, this professional applied local anesthesia with $2 \%$ lidocaine at the intersection of the apex of the triangle formed by the two parts of the sternocleidomastoid muscle in the direction of the ipsilateral nipple [3].
After the administration of local anesthesia, a $30 \times 10 \mathrm{~g}$ needle coupled to a $5 \mathrm{ml}$ syringe with continuous aspiration was introduced using the same technique described above. When the point of the needle pierced the internal jugular vein, the syringe filled with blood [3]. To confirm venous as opposed to arterial puncture, the manometry technique was employed [5]. After confirming the venous puncture, the syringe was uncoupled from the needle, and the body of the needle was blocked with the fingertip to avoid air embolism. Next, the guide wire was introduced through the body of the needle. At this moment, the skin and subcutaneous cellular tissue were dilated with the specific plastic dilator. After dilation, the dual lumen catheter was inserted $\left(\mathrm{Cook}^{\circledR}\right.$, Germany, 2008) to the 15-cm mark $[2,3,7]$.

\section{Technique for US-guided puncture}

The 5 MHz ultrasound machine (Vivid ${ }^{\circledR}$, Helsinki, Finland, 2008) was prepared prior to infiltration with local anesthetic and was placed in a covering of sterile latex with ultrasound jelly protecting the probe. The reseracher performed the puncture positioned at the head of the patient. After dividing the sternocleidomastoid muscle at the medial and lateral potion, the ultrasound probe was positioned and applied local anesthesia with $2 \%$ lidocaine in the direction of the RIJV, which was visualized on the US monitor [2].

After application of local anesthesia, a $30 \times 10 \mathrm{~g}$ needle coupled to a $5-\mathrm{ml}$ syringe under negative pressure was introduced in the direction of the RIJV. When the point of the needle pierced the internal jugular vein, the syringe filled with blood. At this moment, the syringe was uncoupled from the needle, and the body of the needle was blocked with the fingertip to avoid air embolism. The guide wire was introduced through the body of the needle, and the needle was removed. Immediately thereafter, the skin and subcutaneous cellular tissue were dilated with the specific plastic dilator. After dilation, the dual lumen catheter was inserted ( $\operatorname{Cook}^{\circledR}$, Germany, 2008) to the 15-cm mark [2,7]

\section{Outcomes analyses}

Primary outcome: Number of single puncture (counted after local anesthesia was performed; a locating needle was not used [5]);

\section{Secondary outcomes:}

- Puncture duration (the time interval between application of local anesthesia to the skin and insertion of the catheter to the 15-cm mark);

- Incidence of carotid puncture;

- Incidence of changing the puncture site (after three attempts in the RIJV, the site was moved to the right subclavian vein [RSCV]);

- Duration of catheter use; and

- Incidence of infection at the puncture site during the time that the catheter was used.

\section{Statistical analysis}

Parametric and non-parametric data were expressed as the mean \pm $\mathrm{SD}$ and as the frequency or percentage for nominal variables. We used the Student's $t$-tests or Mann-Whitney, according to need, to compare the continuous variables between groups, and the categorical data were analyzed using the Chi-square test. Statistical analysis was performed using the Graph Pad OS5 program for Macintosh, and statistical significance was set as $\mathrm{P}$ values below 5\%. 
Citation: Filho MFS, Lawall P, de Souza KM, Palitot I, Magalhaes I, et al. (2013) Comparison between Ultrasound-Guided and Anatomic Landmark Puncture of the Right Internal Jugular Vein. J Cardiovasc Dis Diagn 1: 128. doi:10.4172/2329-9517.1000128

Page 3 of 4

\section{Results}

Table 1 presents the demographic data for the two groups. There were no significant differences between the groups.

Figure 1 shows the incidence of catheter implantation in a single attempt between US group and Blind group (USG=20 [100\%] and $\mathrm{BG}=12[60 \%] ; \mathrm{p}=0.001)$.

Table 2 presents the clinical outcomes for the two groups. The amount of time required for puncture and for catheter introduction was the same for both groups (USG=119.4 $\pm 56.9 \mathrm{sec}$ and $\mathrm{BG}=137.8 \pm$ $94.6 \mathrm{sec} ; \mathrm{p}=0.98)$. The incidence of carotid puncture was also the same for the two groups (USG=0 [0\%] and $\mathrm{BG}=2[10 \%] ; \mathrm{p}=0.48$ ). Analyzing the incidence of changing the puncture site, the group that underwent US exhibited a lower incidence of changing the puncture site (USG $=0$ [0\%] and $\mathrm{BG}=4$ [20\%]; $\mathrm{p}=0.03$ ). The incidence of infection at the puncture site during the hospital stay was analyzed, and there was no infection at the puncture site for either group.

\section{Discussion}

To minimize complications related to the technique of central catheter implantation, the SCA and the American Society of Echocardiography have defined and established that implantation of a central catheter without employing US is poor medical practice [2].

More than five million central catheters are implanted each year in the United States of America for various reasons, such as hemodynamic monitoring, central venous blood collection, infusion of hyperosmolar drugs, and parenteral nutrition [3]. McGee and Gould reported a series of complications stemming from the insertion of central catheters, in which carotid artery puncture, hematomas at the puncture site, infections at the puncture site, and thrombotic events are the main complications found in adult patients [3].

\begin{tabular}{|c|c|c|c|}
\hline & US Group & Blind Group & p value \\
\hline Patients, $n$ & 20 & 20 & 1 \\
\hline Males, $\mathrm{n}(\%)$ & $13(65 \%)$ & $12(60 \%)$ & 1 \\
\hline Weight, $\mathrm{kg}$ & $75.1 \pm 16.1$ & $72.9 \pm 10.2$ & 0.35 \\
\hline Height, $\mathrm{cm}$ & $170.7 \pm 9.8$ & $166.9 \pm 7.9$ & 0.20 \\
\hline Age, years & $55.2 \pm 5.6$ & $54.4 \pm 7.4$ & 0.98 \\
\hline
\end{tabular}

Table 1: Demographic data for the two groups.

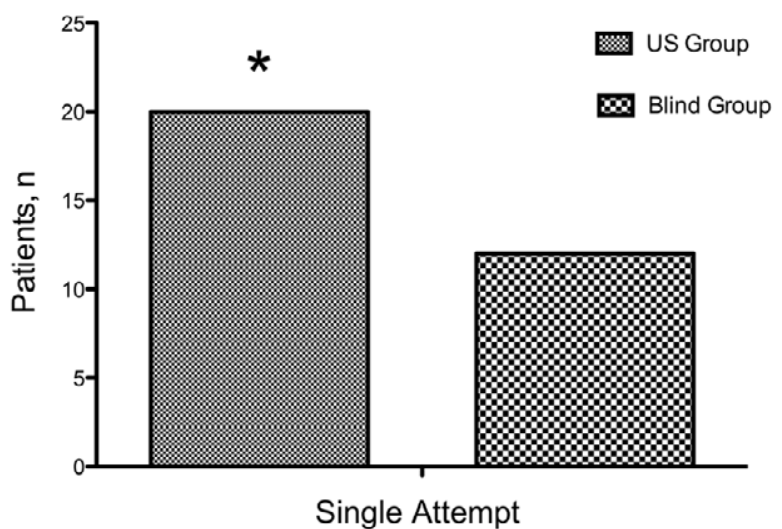

* $p<0.05$ for the Chi-square test

Figure 1: The incidence of catheter implantation in a single attempt between US group and Blind group.

\begin{tabular}{|c|c|c|c|}
\hline \multicolumn{1}{|c|}{ Data } & US Group & Blind group & p value \\
\hline Puncture duration, sec & $119.4 \pm 56.9$ & $137.8 \pm 94.6$ & 0.98 \\
\hline Carotid puncture, $\mathrm{n}(\%)$ & $0(0 \%)$ & $2(10 \%)$ & 0.48 \\
\hline Change of the puncture site, $\mathrm{n}(\%)$ & $0(0 \%)$ & $4(20 \%)$ & $0.03^{*}$ \\
\hline Infection at the puncture site, $\mathrm{n}(\%)$ & $0(0 \%)$ & $0(0 \%)$ & 1 \\
\hline Duration of catheter use, days & $3.4 \pm 1.4$ & $3.3 \pm 1.1$ & 0.98 \\
\hline Hospital stay, days & $8.8 \pm 1.9$ & $10.3 \pm 4.1$ & 0.1 \\
\hline
\end{tabular}

* $p<0.05$ for the Chi-square test

Table 2: Puncture duration and outcomes.

There is a learning curve for performing this technique, which depends on theoretical knowledge of the physical principles of US; knowledge of the operability of the US machine; proper preparation and asepsis of the US probe; knowledge of the superficial anatomy of the puncture site and of the anatomy generated by the US because the anatomical positions often vary; and knowledge regarding pulse, continuous, and color Doppler ultrasound for distinguishing between the artery and vein [2]. Furthermore, training and skill (which vary for each professional) are necessary to simultaneously handle the equipment and perform the puncture, to change the position of the needle in accordance with the anatomy revealed by the US, to confirm the correct positioning after puncture [2].

Given these factors, several authors have reported increased time requirements for performing central venous puncture when it is guided by US. Our results demonstrate that the puncture time for the blind technique and the US-guided technique is the same. When the two techniques are employed in a single puncture, the use of US causes a delay. However, the requirement for more than one puncture in the US group was lower than in the blind group; thus, considering the total duration of the puncture, there was no difference between the groups $[2,4]$.

Troianos et al. [1], McGee and Gould [3] and Bodenham [4] reported a $5 \%$ to $11 \%$ incidence of complications from carotid puncture using blind puncture in their studies. This result is due to anatomical differences in the location of the jugular vein relative to the carotid artery [2-4]. Denys and Uretsky showed that the Internal Jugular Vein (IJV) is located anterolateral to the carotid artery in $92 \%$ of patients, $>1 \mathrm{~cm}$ lateral to the carotid artery in $1 \%$ of patients, medial to the carotid artery in $2 \%$ of patients, and not within the standards for anatomical reference in $5.5 \%$ of patients [8]. In 1998, Gordon et al. defined anatomical relationships between the IJV and the carotid artery in a study that utilized US [2,9].

In our study, the blind group exhibited an $8.5 \%$ incidence of carotid artery puncture, whereas the incidence in the US group was $0 \%$. These findings are in accordance with the literature worldwide, which shows a reduction in the incidence of mechanical complications for patients undergoing US during the insertion of a central venous catheter $[2,5,6,8,9]$

The difficulty of blind central venous puncture is associated with a greater number of punctures and changes of the puncture sites, thus leading to a higher incidence of complications [5,9]. The use of US for central venous puncture reduces the number of attempts and changes of the puncture site because the puncture takes place with direct visualization of the area in which the needle is being inserted $[2,10]$. It is noteworthy that in the USG, $100 \%$ of the catheter insertions occurred on the first attempt, while for the blind group, only $58 \%$ of the cases experienced a successful puncture on the first attempt. Furthermore, no patients from the USG had to undergo changes of the puncture 
Citation: Filho MFS, Lawall P, de Souza KM, Palitot I, Magalhaes I, et al. (2013) Comparison between Ultrasound-Guided and Anatomic Landmark Puncture of the Right Internal Jugular Vein. J Cardiovasc Dis Diagn 1: 128. doi:10.4172/2329-9517.1000128

site; by contrast, in the BG, $23 \%$ of the central venous catheters were inserted into the RSCV. This change of the puncture site occurred in the case of carotid puncture and/or when three punctures were made in the RIJV without success.

One complication that occurs late in the process of central venous puncture is local infection, bacteremia, or even endocarditis and sepsis. These complications may compromise the clinical outcomes of patients who require implantation of a central catheter, thereby increasing their morbidity and mortality $[3,6]$.

With the necessity of reducing the incidence of infectious complications stemming from central venous puncture, many manufacturers produce catheters impregnated with chlorhexidine, silver sulfadiazine, rifampin, and antimicrobials. Randomized and prospective studies have revealed decreased incidences of infections related to central catheters [3]. However, other meta-analyses have not demonstrated the same effectiveness for these catheters, and the aseptic technique that is used for venous catheter insertion is more important [11]. None of the catheters used in the present study was impregnated with antimicrobial substances. The techniques for asepsis and antisepsis were the same for the two groups with the use of disinfecting chlorhexidine and (after three minutes) alcoholic chlorhexidine. Sterile surgical drapes were used, and the anesthesiologist brushed his hands with disinfecting chlorhexidine prior to donning a sterile gown and sterile gloves.

Analyzing the incidence of infection at the puncture site during the presence of the catheter revealed no infection among the groups. In addition to the aseptic technique for the puncture, another factor that might have contributed to the lower incidence of infection was the duration of catheter use. Because all of the patients in this study underwent $\mathrm{CABG}$, the catheter was removed during the transfer from the Intensive Care Unit (ICU) to the ward; thus, the duration of catheter use was reduced in contrast with certain studies in which the catheters were implanted into critical patients who experienced longer stays in the ICU $[3,11]$.

The present study has limitations. For instance, the number of patients was small and the investigation was performed at a single medical center.
Thus, we may conclude that the use of US for the insertion of a central venous catheter in patients undergoing CABG is associated with a lower incidence of vascular complications, a lower incidence of changed puncture sites, and a greater incidence of venous punctures successfully performed in single attempts.

\section{References}

1. Wollmeister J, Conceição DB, Helayel PE, Santos RK (2008) Uso do ultra-som para punção venosa central em paciente obeso com adenomegalia cervical. Rev Bras Anestesiol 58: 403-408.

2. Troianos CA, Hartman GS, Glas KE, Skubas NJ, Eberhardt RT, et al. (2012) Special articles: Guidelines for performing ultrasound guided vascular cannulation: Recommendations of the american society of echocardiography and the society of cardiovascular anesthesiologists. Anesth Analg 114: 46-72.

3. McGee DC, Gould MK (2003) Preventing complications of central venous catheterization. N Engl J Med 348: 1123-1133.

4. Bodenham AR (2006) Can you justify not using ultrasound guidance for centra venous access? Crit Care 10: 175-176.

5. Ezaru CS, Mangione MP, Oravitz TM, Ibinson JW, Bjerke RJ (2009) Eliminating arterial injury during central venous catheterization using manometry. Anesth Analg 109: 130-134.

6. Bailey PL, Glance LG, Eaton MP, Parshall B, Mclntosh S (2007) A survey of the use of ultrasound during central venous catheterization. Anesth Analg 104 491-497.

7. Seldinger Si (1953) Catheter replacement of the needle in percutaneous arteriography. A new technique. Acta Radiol 39: 368-376

8. Denys BG, Uretsky BF (1991) Anatomical variations of internal jugular vein location: Impact on central venous access. Crit Care Med 19: 1516-1519.

9. Gordon AC, Saliken JC, Johns D, Owen R, Gray RR (1998) Us-guided puncture of the internal jugular vein: Complications and anatomic considerations. J Vasc Interv Radiol 9: 333-338.

10. Mallory DL, Shawker T, Evans RG, McGee WT, Brenner M, et al. (1990) Effects of clinical maneuvers on sonographically determined internal jugular vein size during venous cannulation. Crit Care Med 18: 1269-1273.

11. McConnell SA, Gubbins PO, Anaissie EJ (2003) Do antimicrobial-impregnated central venous catheters prevent catheter-related bloodstream infection? Clin Infect Dis 37: 65-72. 